\title{
Tensor-based Multivariate Statistical Discriminant Methods for Face Applications
}

\author{
Jacey-Lynn Minoi \\ Faculty of Computer Science \\ and Information Technology \\ Universiti Malaysia Sarawak \\ 94300 Kota Samarahan \\ Sarawak, Malaysia \\ Email: jacey@fit.unimas.my
}

\author{
Carlos E. Thomaz \\ Departamento de Engenharia Eletrica \\ Centro Universitario da FEI \\ Sao Bernardo do Campo - Sao Paulo \\ Brazil \\ Email: cet@fei.edu.br
}

\author{
Duncan Fyfe Gillies \\ Department of Computing \\ Imperial College London \\ 180 Queen's Gate, \\ London SW7 2RH, UK \\ Email: d.gillies@imperial.ac.uk
}

\begin{abstract}
This paper describes the use of tensor-based multivariate statistical discriminant methods in three-dimensional face applications for synthesis and modelling of face shapes and for recognition. The methods could recognise faces and facial expressions, synthesize new face shapes and generate facial expressions based on the the most discriminant vectors calculated in the training sets that contain classes of face shapes and facial expressions. The strength of the introduced methods is that varying degrees of face shapes can be generated given that only a small number of $3 D$ face shapes are available in the dataset. This framework also has the ability to characterise face variations across subjects and facial expressions. Recognition experiment was conducted using 3D face database created by the State University of New York (SUNY), Binghamton. The results have shown higher recognition rates for face and facial expression compared to the more popular eigenface techniques. The outcome of the synthesis of face shapes and facial expressions will also be presented here.
\end{abstract}

\section{INTRODUCTION}

Human face contains not only information about the identity, gender and age of a person, but also their emotions and intentions. Recognising faces is an innate ability in all human which is done quickly and effortlessly by the brain. Human also have the ability to discriminate accurately the expressed facial emotions for interaction and communication with others. Even though humans have acquired powerful capabilities of language, the role of facial expressions in communication remains substantial. Interestingly, a duality exists in human recognition making recognising the different facial expressions of an unfamiliar person and recognising a familiar person regardless of the generated facial expressions possible and easy. However, this interface is still far from having it to be automatically computerised.

There are numerous research on developing tools and methods for face applications. Despite the maturity of research work among face community, there is not yet a face application that can deal with the duality of automatic face recognition and facial expression recognition. In the current literature on automatic face recognition, many researchers often use the same techniques deployed on face recognition to facial expression recognition and other face applications. There are a number of face variants, for example the differences arising from age, gender, pose, illumination, illness, growth or shaving of beards or facial hair, make-up and facial deformation due to speech, which could influence the accuracy of face recognition as they are unwanted to the uniqueness of the face.

To account to this issue, we could create subspaces for each of the face variation and establish relationships among the subspaces. Here, we are looking at using a more advanced algebraic and statistical framework by introducing tensorbased multivariate statistical models to represent the threedimensional face models into a parsimonious fashion. A commonly used statistical discriminant technique, Principal Component Analysis (PCA), can be used to model variations, but it will not separate these different sources of face variants. A tensor model could be viewed as a multilinear representation of a set of data and hence it allows the manipulation of data independently on multi-dimensions of change. The modes represent different variations after performing decomposition with multiple formation factors and these factors are face variations. Details of the tensor model can be found in Tucker [15] and Lathauwer [10]. We chose to apply Singular Value Decomposition (SVD) method on the modes for its uniqueness and assured orthogonality of the matrices.

Our research looked into representing faces and developing face applications using a multilinear algebra technique based on higher-order tensors. A tensor is a structure generalising vector spaces. Higher Order Singular Value Decomposition (HOSVD) will be used to manipulate the tensor model. HOSVD is a general $N$-factor analysis method. Vasilescu and Terzopoulos [1] used HOSVD in 2-dimensional(2D) image face recognition. Further work by Macedo et al. [4] followed Vasilescu and Terzopoulos but used a different set of data. Wang and Ahuja [2] worked on synthesizing facial expressions based on the results gathered from HOSVD. Vlasic et al. [3] applied a tensor framework to synthesize facial expressions. The results of these research have proved that tensor-based multilinear approach is promising in handling multiple independent face variant factors simultaneously.

Research in the area of face recognition has been complemented by interest in facial expression research. Synthesizing facial expressions to portray emotions, analysis of facial 